\title{
Energy-using products as embodying heterogeneous requirements
}

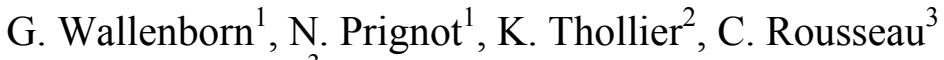 \\ \& A. Van Cutsem ${ }^{3}$ \\ ${ }^{1}$ Université Libre de Bruxelles, Belgium \\ ${ }^{2}$ Institut de Conseil et d'Etudes en Développement Durable, Belgium \\ ${ }^{3}$ Centre de Recherches et d'Information des Organisations de \\ Consommateurs, Belgium
}

\begin{abstract}
This paper presents a newly founded research project that is conceived as an integrated socio-technological study about the manufacturing and use of household energy consuming appliances (often referred to as EuPs). This project will examine how standards and ecodesign could take into account the different kinds of users and their role in the use phase in terms of energy consumption. The paper presents the motives to study the use phase of EuPs. It presents these products as embodying conflicts and trade-offs of constraints pertaining to different categories of requirements: technology, economy, ergonomics, social and cultural aspects, health and safety, ecology, ethics. What do EuPs tell us about a culture of energy that is needed for a sustainable development?

Keywords: energy-using products, eco-design, ecological standards, users.
\end{abstract}

\section{Introduction: a new culture of energy is required}

This paper is the starting point of a new research about the ecodesign of energyusing products (EuPs). In our research, we shall conceive these products as a conjunction of different domains of practices: standards of different kinds, conception and construction process, users, policies, market. All these domains of practice are obviously interconnected through the analysed products. Practices that are occurring in these domains precisely define them: some people take time to build standards, others try to achieve proper legislation, while some make their best to provide nice and cheap products that some others have conceived, 
designed and constructed. But everybody (at least in countries which have reached a certain level of economic development) uses on a daily basis objects that require networked energy.

There are many reasons to be fascinated by objects that operate with readymade energy. They are simply startling: electrical and electronic appliances are pure magic, enchanted by the Fée Electricité. They have shaped our society and the way people work, cook, wash, are entertained, communicate, etc. When combined with a heating system, they are modern marvels.

While these impressive objects are being constructed and sold, their number is becoming tremendous. Electricity consumption is steadily increasing in the OECD [1], due to a.o. an increase of the penetration rates of electrical appliances in households and of their use, as well as the appearance of new apparatus and new functions. As Elizabeth Shove [2] shows, standards of comfort have risen and are still rising. This is often referred as "the rebound effect" in sociology. This trend is expected to continue while society undergoes socio-demographic change: the decreasing average size of households will entail more energy consumption per capita; the increasing mean age will raise comfort standards.

Today everybody eventually acknowledge the necessity to reduce energy consumption, for various reasons. Global climate change is at the forefront of preoccupations now, but petrol depletion and energy independence are also important. In fact the global agreement seems to converge on the necessity to reduce carbon dioxide emissions in the atmosphere. Different means are possible to achieve this goal: improve energy efficiency, develop renewable energies, sequester carbon; for some people: advance nuclear fission (or fusion), reduce the number and the use of apparatus possessed by households.

We have observed in a previous research that at least in Belgium, a new culture of energy seems to be required anyway: we know some parameters of the new equation though that is not sufficient to make decisions. In the current culture, energy is abundant, cheap and invisible. In the upcoming culture, energy is fragile (think about blackouts), complicated (multiplication of sources and providers), more climate damaging and more expensive.

\section{Framework of the study}

We have observed that in the current culture of energy (at least in Belgium), people are not guided by one single energy use rationale or one single energysaving rationale [3]. Their rationales tend to depend rather on the sector of household activity, i.e., lighting, heating, cooking, washing clothes, entertainment, etc. People make choices and adopt certain behaviours in each of these sectors in line with a set of criteria and constraints in which saving energy or money is often a less important factor than other personal criteria. Motivations sustaining practices vary from one person to another, and even vary for the same person from one category of appliance to the other, in such a way that it is not possible to define a standardised consumption practice that would apply for everybody or for all categories of appliances. In other words, people's behaviours cannot be explained only by a lack of information: sectors are 
specific to uses and practices rather than to energy. Energy gets presence only through its various uses, as heating, lighting, cooking, washing, etc. Energy is an irrelevant concept from the point of view of practices. Energy is an abstract term for the common people. It is without perception: energy is not associated to the practices that lead to its consumption. It appears only through its manifestations. Relation to energy is both.

Related to the problem of energy consumption in households, the European Union has launched a legal framework for improving the environmental impacts due to energy using products [4]. EuPs cover a wide variety of products: they range from white appliances to heating devices, including central heating, as well as a series of smaller equipment with a focus on stand-by and off consumption. This regulation will force EuPs to conform to a series of standards in order to be on the European market; these standards will be based on a series of assessment studies of the different products that have begun in 2006. This new regulation and the stakeholders forums appear to be an interesting ground to be investigated as it will set the level of requirement for the concerned appliances. We will thus use the same product categories (restricted to the products commonly found in households) in order to start our project: boilers and a series of electrical appliances ranging from white appliances to lighting.

Moreover, our previous research has shown that consumers' behaviours in their home environment are diverse, and are marked by specific beliefs and habits [3]. This diversity of habits and beliefs is mostly neglected in ongoing policies (standardisation, ecodesign of products), where consumers are relied on in order to make rational and efficient choices. To avoid the fallacy of rational choice theory, the rationality of consumers' choices must always be situated, that is, placed in the context of the constraints that limit their theoretical possibilities of action. We will then analyse these aspects and therefore add a specificity neglected by the directive.

Studies about technology often stop at the threshold of the use phase or assume a standardised behaviour, and studies about energy consumption neglect the way users are shaped or influenced by appliances. In research, development and standardisation phases, the user is considered as an average user acting rationally. Technical objects are rarely taken in their activities, in what they are enabling. That is rather paradoxical for they are constructed for acting. But this activity seems to appeal few theoretical analysis: too daily, too routine, too obvious, too wont. Besides, it is important to note that this enablement is constructed in shaping uses and in hindering some other uses. Uses of an object are fixed activities (or a range of fixed activities) that have been designed.

How are these fixed activities constructed? How do the designers think of the lay user? Does their concretisation match forecast made by designers and producers? How are these objects utilised by users? These are some of the questions on which our research wants to bring some light. The project will explore and analyse the relationships and articulations around some energy-using products, namely among designers, producers, retailers, buyers and users. Each appliance will be examined from different points of view: LCA, engineer, designer, marketing, retailer, user, repairman, ... Every actor has another view 
about the appliance and its use. These views probably differ too among the same category of actors. We assume that some misuses arise from divergent views about use by different actors. We then will explore representations that users have, as well as representations of other actors about users and uses. But we will not neglect the central actors of our research: in the framework of sustainable development, what kind of new culture of energy is required from the point of view of the users and of the objects that require energy?

In the following sections, we shall first show the importance, though often neglected, of the use phase of energy-using appliances. We shall then sketch a typology of the different requirements that weigh on household energy-using products.

\section{Why focusing on the use phase?}

In the articulations around energy-using objects, the interface between a user and an object is peculiar. Of course, each articulation is singular since it talks from the point of view of a specific practice. From a LCA perspective however, the use phase is generally the least known of the different phases (design, extraction, manufacturing, assembly, distribution, use, disposal). The production phase is now extensively known and databases exist that allow a computation of production impacts on the environment, even if the assembly step is less extensively known. On the contrary, the assessment of the use phase is mostly done on the basis of averaged behaviours collected by different studies, not to mention the numerous "expert assumptions" for data which are still missing. The impact of real consumers' behaviour is thus seldom taken into account (or roughly) in LCAs, mostly because it is not yet fully known, or at a very aggregate level which does not allow differences of uses to show up. The importance of use phase is particularly obvious in the case of the management of the heating system, where the settings (or their absence) of the regulating system is one of the key factors of energy consumption in a house.

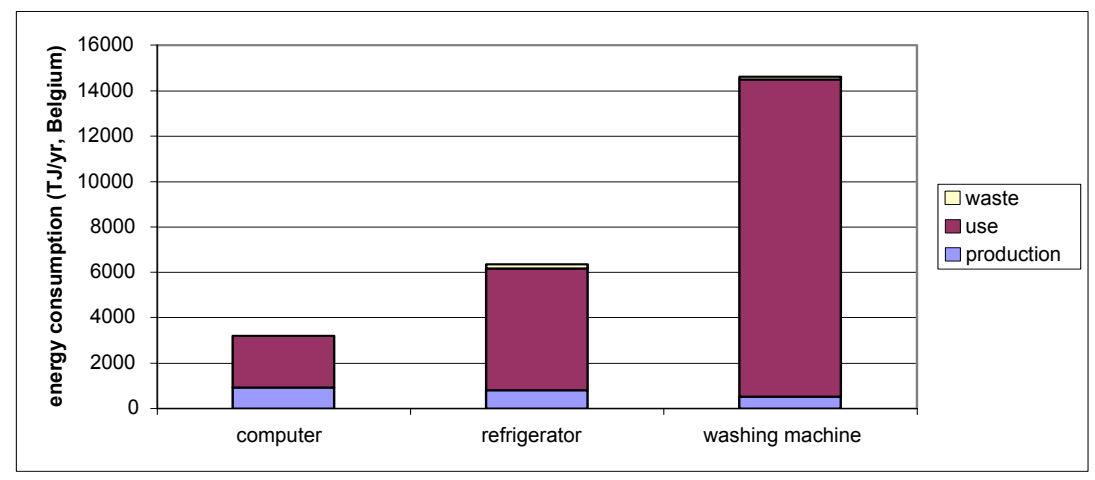

Figure 1: $\quad$ Energy consumption by phase for 3 EuPs. 
On another hand, the largest impact of an EuP on the environment occurs during its use phase, compared to the production phase [5], as one can see in the figure 1. Only in the case of computers is the energy consumption in the production phase more important (about $1 / 3$ of total energy consumption over life cycle) [6]. In most cases, the production phase has very few impacts compared to the use phase. It should be underlined, however, that the rising use of chips and other electronic devices in every electrical equipment increases the amount of energy required to manufacture the appliances (embodied energy).

In the current culture of energy, users are defined as passive and ignorant from the point of view of energy consumption. So the pedagogical model of 'the blackboard' is used. Discourses about rational use of energy are typical of this kind of professorship. You just teach people as if they were in a school. Yet how could people become active? What would be a good practical lesson?

In the current culture, energy is invisible. How would it be possible to make it visible? In our previous study, we have thus observed that energy consumption dynamics in a household are totally different when considering the domestic sectors. People seem moreover much more reluctant to change their behaviours than to acquire new equipment. A logical conclusion is that it would therefore be easier to rely on appliances to change habits and practices. $69 \%$ of Belgian people state that they would pay attention to energy consumption if their appliances displayed this consumption. $57 \%$ find that a software managing their energy consumption from their computer would be useful.

We have observed in focus groups that some people are willing to know more about their instant energy consumption, and even to be "educated" by appliances. Could users be educated by the appliances? User's guides are generally poorly written. In most cases, they are not read by users who seem to prefer a direct confrontation with the equipment. Technical objects can embody morality, as the beep of the safety belt when not tied up [7].

Our approach will seek to overcome the classical dichotomy between technology and culture. Whom should we expect change from, appliance performance or user's behaviour? We think we have to decompose objects into its different elements (and constraints) which are both technological and cultural. A conceptual tool to decompose objects is the study of controversies about their construction. Controversies are indeed events in which it is possible to grasp how interests of different actors shape appliances. Interests are intertwiningly both economical and technical. Another conceptual toot is the exploration of tensions among different points of view about an object.

\section{Appliances as embodying conflicts and trade-offs between different requirements}

The project aims at exploring how standards and ecodesign could take into account the use phase and the different kinds of users. The socio-technical context in which objects are constructed is very important, as the cultural context is. Questions around dematerialisation, around consumption society and the act of buying, are very interesting. However, besides considerations about the 
context in which objects are constructed, distributed, bought and used, we would like to sketch a typology of conflicts between different requirements that shape objects.

Indeed each appliance embodies a series of constraints pertaining to different categories of requirements: technology, economy, ergonomics, social and cultural aspects, health and safety, ecology, ethics,... We shall explore these constraints and how they are mutually negotiated for eventually constituting an appliance. The result of this analysis should show which constraints could be improved in a sustainable perspective. In other words, our study aims at assessing the actual room for manoeuvre in standard elaboration in order to ease energy demand side management in households.

EuPs can be seen as an agency of constraints of different kinds that can be categorised as follows:

- technological: the product must work; it has to follow the engineer's rules; electronics plays an increasing role in management devices;

- economical: the product ought to be sellable and profitable; it has then to be competitive;

- ergonomic: the product is required to be user-friendly;

- social and cultural: the object should meet a function or a desire; it must conform to transitory esthetical standards;

- health and safety: the object must meet safety standards ; it cannot contain hazardous substances that could be disseminated;

- environmental: the object is made from available raw materials; standards may impose limits to environmental impacts;

- ethical: the product should respect rights of the workers who built it.

Would it be possible to produce appliances that would be user-friendly, with a low energy consumption, with a long lifetime, recyclable, cheap... Are some requirements contradictory? If so, how are the priorities set? By whom? In which LCA phase should and could standardisation be carried out? Which standards are to be implemented? It is then essential to analyse the different categories of constraints that shape appliances. Bearing in mind that controversies and conflicts are good revealers of agency of objects that appear obvious, we will consider conflicts that can arise between different requirements. This list is obviously exploratory and not exhaustive.

\subsection{Conflicts and trade-offs arising at the conception phase}

\subsubsection{Safety and environment}

Many appliances consume electricity even when they appear to be turned off, because they contain transformers that are still connected to the power supply. When an appliance embodies electronic devices, a transformer is required to put adequate voltage in the chips. The interrupter is placed on the secondary circuit of the transformer, for sparks are less dangerous at $12 \mathrm{~V}$ than at $220 \mathrm{~V}$. But electricity continues to flow in the primary circuit resulting in consumption even in the "off" mode. 


\subsubsection{Cost and environment}

Related to the first example mentioned above on safety and environment, bigger switches can be found which could be placed on the primary circuit of appliances. They have to be designed especially to prevent sparks at $220 \mathrm{~V}$ and are thus slightly more expensive than the $12 \mathrm{~V}$ switches. The difference is not large when compared to the cost of the equipment, but is sufficient to prevent manufacturers from using them, since there is no clear demand for this change.

\subsubsection{Environment and usability}

The standby problem is well known: the embodiment of secondary functions (clock, remote control, light token, ...) stands for approximately $10 \%$ of household electricity consumption. Some advices tell people to buy a socket with a hard switch to allow them to completely switch off the electric appliances. We have however observed that few people are willing to do so. It seems then, if people want to keep the possibility to switch off from their armchair that the solution could come from chips that consumes 0.1 Watt or less.

Another linked problem lies in "set-top boxes" or decoders. These objects are continuously functioning and users are recommended not to switch them off. Fans and light in a fridge are another example of the conflict between usability and reducing energy consumption, since they contribute to heating the fridge.

\subsubsection{Safety and health}

Another example is the use of fire retardants in most EuPs. These retardants are required in order to prevent the burning of the plastic cases of TVs, monitors, etc in case of electrical fire. However, the brominated substances used for that purpose are far from being harmless. Moreover, the plastic degrades slowly due to the heat (during use) resulting in a harmful dust which is found and breathed around these appliances (see for example [8]).

\subsubsection{Source of energy}

It is sometimes possible to conceive a product that can use gas instead of electricity (dryers). Solar energy can sometimes be directly used in the following objects: calculator, torch, garden light, ... Mechanical energy can be used for some objects: watch, radio, torch.

\subsubsection{Ergonomics}

It seems that a lot of new appliances come back to the retailers in the first weeks after their purchase, while they are perfectly working. Appliances are often too complex for the average user. However, this complexity may not be adequately oriented: for example we have observed a demand for a screen indicating the energy consumption. What are the reasons for the increasing complexity of EuP? Would it not be interesting to put on the market basic models with simplified functionalities?

\subsubsection{In different phases}

It is possible to install electronic devices that reduce consumption in providing feedback information but pollute when are produced and discarded. The expected outcome in terms of reduced energy consumption should be studied. 
Compact fluorescent bulbs are much more energy efficient than incandescent light bulbs. They allow also one to make resource conservation since they have a longer lifespan. However, their disposal has to be carefully done for they contain hazardous substances.

\subsection{Conflicts and trade-off when purchasing}

The zero-point of purchasing conflicts is about the very fact of purchasing: Is the object needed? Members of a household can disagree about that issue.

\subsubsection{Cost and environment}

Eco-efficient appliances are generally more expensive when bought than less efficient ones. Therefore, their access is limited to household able to make the investment, even though these appliances are cheaper when considering both purchase and consumption prices.

\subsubsection{Comfort and environment}

Mechanical appliances are replaced by electric ones: can opener, toothbrush, high pressure cleaner, ...

\subsubsection{Design and style narrow the choice}

When appliances have to be built-in, the choice can be restricted. The desire for fashionable objects (e.g. 'American' fridge), or simply for their style or colour can lead people to purchase high consumption objects.

\subsubsection{Perceived performance and energy}

For some products, the market demand is for greater energy consumption where power is falsely considered to result in better performance (e.g. vacuum cleaner).

Also, for computers, people tend to buy fast equipment with several peripherals (DVD players, etc) while they do not have the use of this equipment.

\subsection{Conflicts when using appliances}

\subsubsection{Optimisation}

Appliances are optimised for operation at full load, yet this condition rarely occurs (be it for washing machines, heating systems, power supplies etc). Often such systems are inefficient at partial load. Furthermore, appliances are optimised in laboratory that can be far away from effective use (e.g. fridges that are run in poorly ventilated rooms).

\subsubsection{Hygiene and environment}

For hygienic reasons, people can wish to wash their clothes at high temperature $\left(90^{\circ} \mathrm{C}\right)$ or rinse them abundantly. They can also set the temperature of the deepfreezer at extremely low temperatures, less than the recommended $-18^{\circ} \mathrm{C}$, in order to slow food decay. 


\subsubsection{Rational use of energy}

Some uses of energy cannot be "rationalised", for they are a-rational. For instance, lighting is used for creating space, creating presence in non occupied rooms, creating safety outside the dwelling or simply outlining decorative fixtures. Therefore the advice to switch lights off is irrelevant.

\subsection{Conflicts when an object is broken}

Durability or efficiency? An appliance designed for a longer life reduces both use resource and waste generation. However the improvement in energy efficiency makes the replacement at some time interesting for some equipment that is consuming less energy now than before. But when should an appliance be replaced? How can one compare resource use and waste to energy use?

Technological progress seems to be planned in order to upgrade the market on a regular basis, and therefore to propose new appliances to consumers. Objects are sometimes discarded while still functioning because they are note fashionable anymore. The trend today is to buy and dispose of more (and if possible cheaper) appliances, replaced at a relatively high pace. How would it be possible to restore reparation or upgrade as a viable alternative? What are the constraints for repairing or upgrading EuP today? Relative prices of buying versus repairing seem to be the main determinant to buy a new appliance $[9,10]$.

\subsection{Controversies about technological future}

Some manufacturers assert that the energy efficiency of some (large) appliances is now close to the optimum and that further improvement would be difficult to achieve, if technology does not change. That is contradicted by some authors (Fawcette quoted in [10]).

Durability means allowing one to repair (possibility and availability of parts). An alternative would be to design appliances for upgradability. Would it be possible to construct objects that can be improved and not just thrown away?

In the framework of a new culture of energy, what would be a new aesthetic that support long term use?

\subsection{Conflicts of policy}

Producers say that they make their best in order to put efficient products on the market, and add that consumers do not always follow. They call then public authorities in order to enforce new rules. The "ecodesign directive" summon producers to produce information and ask consumers to actively search and read this information. Most users, however, are not likely to behave this way.

\section{Conclusions}

To be put on the market, an appliance must meet legal norms, notably safety standards. Producers have interest in marketing desirable objects, working properly for the expected use, at an attractive price but allowing one to make 
profit. Therefore, if there was no legislation imposing a lower energy consumption or requiring a lower impact on the environment, ecological standards would not be a primary issue for producers. Ecological standards add on other criteria and materialise new conflicts and necessary trade-offs.

At first glance, conflicts arise mainly in the conception phase and in a lesser extent at the purchasing point. That does not mean however that use phase is negligible, for we have seen that use phase is the most impacting phase. It is then of paramount importance that new regulation take the different kinds of users into account.

In order elaborate further this research, we will have to rework the general categories of economics, safety, performance, environment, ergonomics, policy, etc. in order to fit in the different conflicts that can arise when constructing an energy-using object. Indeed, these categories appear too wide to explain precisely the different conflicts around energy-using objects.

\section{Acknowledgement}

The study is funded by the Belgian Science Policy Office in the framework of "Science for a Sustainable Development"

\section{References}

[1] Waide P., Lebot B., Harrington P., The historic and potential impact of residential electrical equipment energy efficiency policies in the OECD, 2003.

[2] Shove E., Comfort, cleanliness and convenience: the social organization of normality, Oxford, Berg, 2003.

[3] Wallenborn G., Rousseau C., Thollier K., Specifying household profiles for more efficient energy demand-side management, Final Report, Belgian Science Policy, 2006.

[4] Directive 2005/32/EC of the European Parliament and of the Council of 6 July 2005 establishing a framework for the setting of ecodesign requirements for energy-using products.

[5] Nemry F., Thollier K., Jansen B., Claeys P., Hijit M., Priority policies and measures for the federal product policy in order to mitigate climate change, Final report, for the Belgian Federal Services for Environment Climate Unit, 2004.

[6] Thollier K., Jansen B., Reducing life-cycle impacts of housing and computers in relation with paper, ERSCP conference proceedings, 2005.

[7] Latour B., La clef de Berlin, La Découverte, Paris, 1993.

[8] Birnbaum L., Staskal D., Brominated flame retardants: cause for concern?, Environmental Health Perspective, volume $112 \mathrm{n}^{\circ} 1,2004$.

[9] Lewis H. \& Gertsakis J. (eds.), Design + Environment, Greenleaf Publishing Limited, Sheffield UK, 2001.

[10] Michaelis L. \& Lorek S., Consumption and the Environment in Europe. Trends and Futures, Danish Environmental Protection Agency, 2004. 